\title{
Expression of Chirality in Tailor-made Conjugated
}

\section{Polymers}

\author{
Marie-Paule Van Den Eede ${ }^{1}$, Lore Van Gestel ${ }^{l}$ and Guy Koeckelberghs ${ }^{1 *}$ \\ ${ }^{1}$ Laboratory for Polymer Synthesis, KU Leuven, Celestijnenlaan 200F, B-3001 Heverlee, \\ BELGIUM
}

Keywords: Conjugated block copolymers, Pd-RuPhos, supramolecular organization, chirality, circular dichroism, chiral expression

\begin{abstract}
In this manuscript the supramolecular organization and chiral expression in tailormade conjugated polymers $(\mathrm{CP})$ is investigated. The polymers were synthesized in a controlled way, providing (almost) perfect control over the molecular structure. This allowed to understand the effect of different parameters in the molecular structure. The polymers were analyzed by gel permeation chromatography (GPC) and ${ }^{1} \mathrm{H}$ NMR. Solvatochromism experiments were performed on all polymers, using UV-vis spectroscopy and circular dichroism (CD). Two new parameters influencing the supramolecular organization and chiral expression were found. First, in block copolymers consisting of an $(S)$-chiral and $(R)$-chiral block of equal length, end-groups are required to break the symmetry. If no end-group is present, the polymer chains do not aggregate. Second, if one single $\mathrm{HH}$-coupling is present in regioregular poly(thiophene), the supramolecular aggregation is completely disrupted, which in contrast to the presence of one TT-defect. The obtained results were confirmed by differential scanning calorimetry (DSC).
\end{abstract}




\section{INTRODUCTION}

Chiral molecules i.e. molecules with non-superimposable mirror images or enantiomers, are crucial for the creation of molecular recognition, selectivity, and for the creation of systems used in novel applications. ${ }^{1-3}$ Although chemical synthesis without special intervention results in racemic mixtures, the presence of single-handed molecules in life is no exception. More in particularly, almost all chiral molecules in living organisms are found in only one single form. Typical examples are the right-handed sugars and the left-handed amino acids. Understanding the presence of this homochirality in life, remains an unsolved major question.

Although the presence of chirality can easily be checked using symmetry parameters, expression of chirality is much more difficult to understand and control. Expression of chirality in polymers typically arises from the formation of a macromolecular (i.e. helix) or supramolecular (helical stacks) structure. Therefore, fully understanding the chiral expression and more specifically, the molecular parameters which can have a subtle influence on this expression, is of great interest. Chiral expression has already been studied in different systems and at different scales. ${ }^{4-9}$ Those investigations form an important step to understand the chiral expression from nano to macro scale. Here, we want to study the chiral expression and supramolecular organization of poly(thiophenes) (PTs). ${ }^{10-16}$ The reason for this is double. First, poly(3-alkylthiophene) (P3AT) with a chiral alkyl side chain gives rise to a supramolecular structure with strong chiral effects. ${ }^{17,18}$ Second, these polymers can be synthesized in a controlled way, providing (almost) perfect control over the molecular structure. This allows to understand the effect of every single parameter. 
When such chiral PTs organize, right-handed or left-handed macromolecular helices are formed, visualized by UV-vis and circular dichroism spectroscopy. It is generally believed that the chiral expression of regioregular head-to-tail (HT) P3AT is well understood. Nevertheless, although most of those P3AT are prepared from a $\mathrm{Ni}^{+\mathrm{II}}$-salt (e.g. $\mathrm{Ni}(\mathrm{dppp}) \mathrm{Cl}_{2}$, dppp: 1,3bis(diphenylphosphino)propane) the presence of the inevitable tail-to-tail (TT) defect is typically overlooked. Even more, it's location at the beginning of the chain (in case of unidirectional growth) or somewhere in the middle (in case of bidirectional growth) has never been taken into account, although this must effect the supramolecular organization and hence, the chiral expression.

Chiral expression in block copolymers is even more challenging and it also gives the opportunity to evolve towards a system that is nearly symmetric, but still chiral. Our research group expressed chirality in a block copolythiophene that did not contain any excess of chiral centers, but where the evoked chirality was only a result of a specific order of events. ${ }^{18}$ However, apart from the $(S)$ - and $(R)$-chiral monomers, also achiral monomers were built-in, necessary for the creation of the specific order of events. Later, a block copolythiophene of which the first block consists of only $(S)$-chiral monomer and the second block of only $(R)$ chiral monomer (polymer P0, figure 1) was prepared. ${ }^{19}$ Although no excess of chiral monomers was present, and both monomers behave in the same way, with exception of their chirality, a very strong chiral response was obtained. This was explained by the presence of the end-group at the beginning of the first $(S)$-chiral block. Although the incorporation of the end-group is necessary from a synthetic point of view (in order to exclude bidirectional growth), it creates a difference in solubility between both blocks and gave the $(R)$-chiral block the ability to establish its handedness first, and to impose this onto the $(S)$-chiral block. 


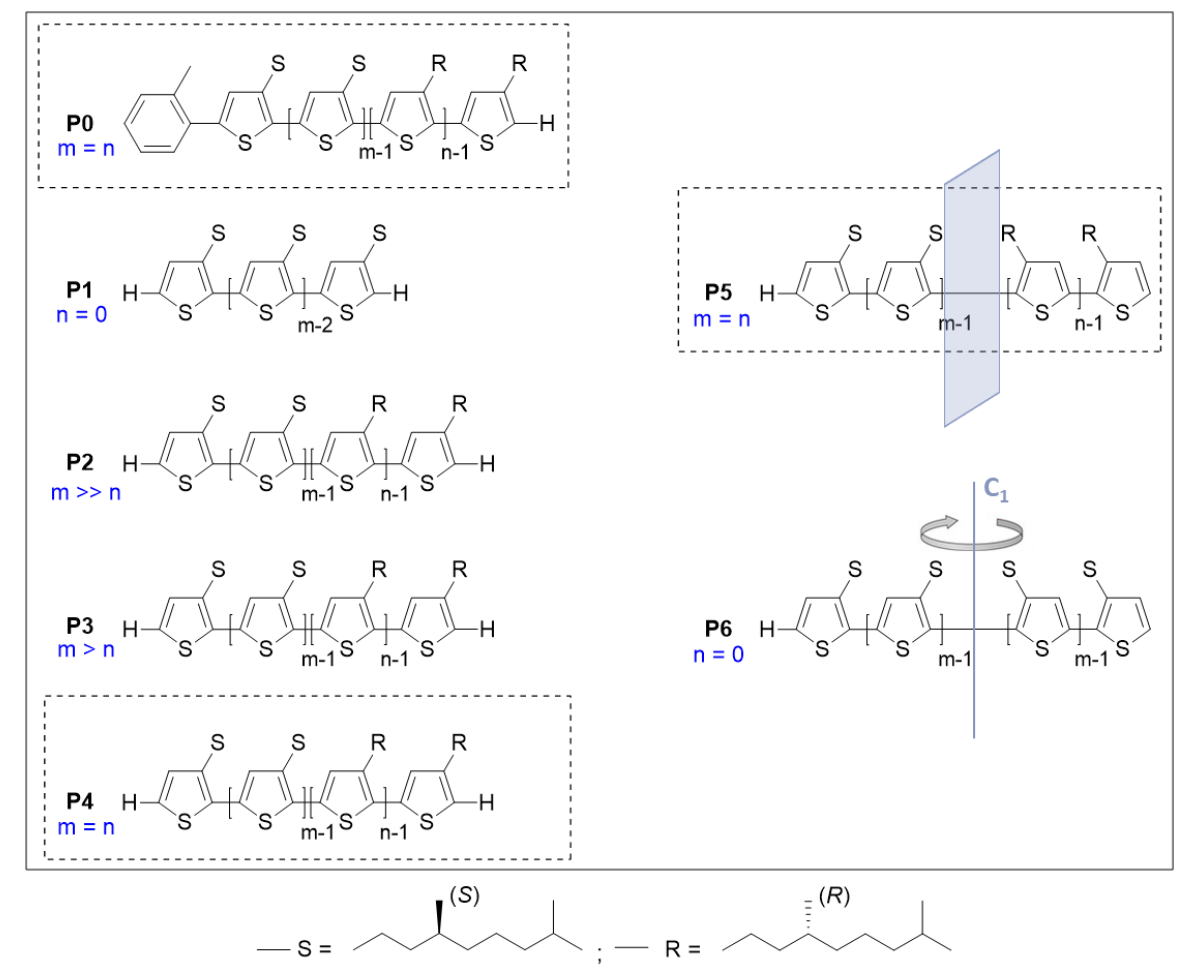

Figure 1. Schematic representation of polymer P0-P6.

In order to exclude the influence of the end-group polymers P1-P4 are designed. These polymers consist of a first block of only $(S)$-chiral monomer and a second block of only $(R)$ chiral monomer, but where, in comparison to the previously synthesized $\mathbf{P 0}$ that has an $o$-tolyl end-group, ${ }^{19}$ also the influence of an different end-group has been removed. However, P4 with exact the same amount of $(S)$ - and $(R)$-chiral monomer, is still not symmetrical: the substituents are all in the 3-position and both ends of the polymer chain are different. Therefore P5, which contains a mirror plane, is created (Figure 1). This mirror plane makes P5 an achiral system. However, it also introduces a head-to-head $(\mathrm{HH})$ defect. In order to investigate the influence of this single defect, also the homo $(S)$-chiral P6 is synthesized as a reference. The absence of an improper rotation axis $\mathrm{S}_{\mathrm{n}}$, makes polymer $\mathbf{P 6}$ chiral (Figure 1). 


\section{RESULTS AND DISCUSSION}

Monomer synthesis. As the polymers will be prepared using a Negishi reaction (see further), the formation of polymers P1-P6 requires the synthesis of 4 different monomers (Figure 2). $(S)$-chiral monomer 1 and $(R)$-chiral monomer 2, possess the iodo-group on the 5-position, while $(S)$-chiral monomer 3 and $(R)$-chiral monomer 4 , on the 2 position. These last two monomers are necessary for the synthesis of the second block of P5 and P6.

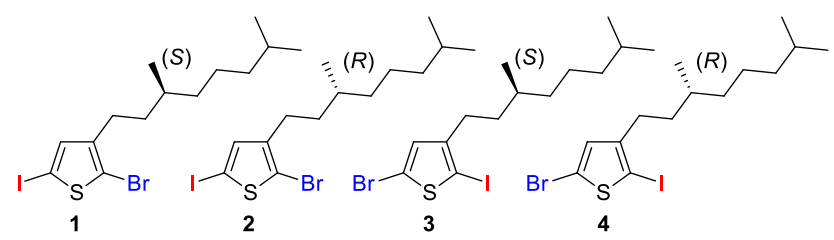

Figure 2. Schematic representation of monomers 1-4.

Chiral monomers 1-3 were prepared according to literature. ${ }^{20,21}$ However, $(R)$-chiral monomer 4 was prepared for the first time (Scheme 1). For this synthesis, 4a was deprotonated in the 5position using in situ prepared tetramethylpiperidinyl lithium and quenched with $\mathrm{CBr}_{4}$. After purification, compound $\mathbf{4 b}$ was obtained as a colorless oil. In a last step, $\mathrm{I}_{2}$ and $\mathrm{PhI}(\mathrm{OAc})_{2}$ were added to a solution of $\mathbf{4 b}$ in dichloromethane, which resulted after purification in monomer $\mathbf{4}$.

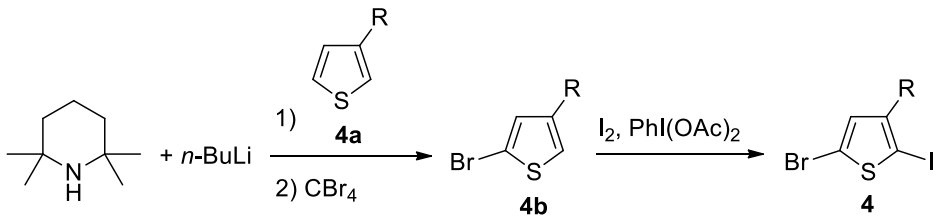

Scheme 1. Simplified representation of the synthesis of monomer 4.

Polymer synthesis. For the synthesis of P1-P6 different requirements need to be fulfilled. First of all, a polymerization mechanism is needed that does not incorporate an end-group different from the monomer unit of the first block. Since the Kumada catalyst transfer condensative polymerization (KCTCP) of thiophene block copolymers with the standard external $o$-tolyl 
initiator, results in block copolymers with an $o$-tolyl end-group, as was the case for $\mathbf{P 0}$, another external initiator is needed. ${ }^{22-26}$ Since the external $\mathrm{Ar}-\mathrm{Ni}\left(\mathrm{PPh}_{3}\right)_{2} \mathrm{Br}$ in which the aryl group is the $(S)$-chiral thiophene unit was impossible to isolate, and since for polymers P5 and $\mathbf{P 6}$ a HHcoupling needs to be formed in the beginning of the second block, another polymerization mechanism is needed. KCTCP based on $\mathrm{Ni}(\mathrm{dppp})$ or Ni(dppe) catalyze only very slowly $\mathrm{HH}$ couplings (much slower than HT-couplings) ${ }^{27}$, which would result in a mixture of homopolymers of the first block and diblock copolymers with a very long second block. However the Pd-RuPhos polymerization, based on the deactivation of the monomer, was developed. ${ }^{28}$ The external initiator $\mathbf{5}$, as shown in scheme $2 \mathrm{~b}$, has already successful been prepared with the $(S)$-chiral thiophene as aryl group. Furthermore, it has also been demonstrated that HH-couplings can easily been formed (the rate constants of HH- and HTcouplings are similar). ${ }^{21}$ As such, this is the ideal polymerization mechanism in order to form all the polymers. The origin of the controlled nature of this polymerization is the deactivation of the monomer. Indeed, the catalyst can dissociate from the growing polymer chain - in contrast to CTCP - leaving a Br-terminated chain. Reinsertion results in further growth. Since this Pd-RuPhos polymerization is sensitive to moisture, very dry conditions are required. Indeed, in the case monomer is protonated, transfer reactions will take place, decreasing the quality of the resulting polymers. An overview of the polymerization is visualized in scheme 2. Prior to polymerization, precursor monomers 1-4 were dried under vacuum over $\mathrm{P}_{2} \mathrm{O}_{5}$, and $\mathrm{ZnBr}_{2}$ at $200^{\circ} \mathrm{C}$ and under vacuum. In the polymerization, a solution of precursor monomer $\mathbf{1}$ in dry THF underwent a Grignard metathesis (GRIM) reaction with $t$ - $\mathrm{BuMgCl}$ for 60 minutes, after which 1 a reacted for 15 minutes with a solution of $\mathrm{ZnBr}_{2}$ in dry THF, thereby forming the organozinc monomer $\mathbf{1 b}$. Subsequently, $\mathbf{1 b}$ was added to a dry THF solution of initiator $\mathbf{5}$ and polymerized for 50 minutes. Termination of this polymerization with acidified $\mathrm{THF}(\mathrm{HCl}$, 1M) resulted in polymer P1. For polymers P2-P4 predetermined quantities of precursor 
monomer $\mathbf{2}$ were transformed into the corresponding organozinc compound $\mathbf{2 b}$, in the same way as precursor monomer 1 , after which this was added to the first $(S)$-chiral block. After 50 minutes, also these polymerizations were terminated with acidified THF $(\mathrm{HCl}, 1 \mathrm{M})$, resulting in polymers P2-P4. For polymer P5 and P6 the same procedure was followed as for P2-P4, but with respectively precursor monomers $\mathbf{4}$ and $\mathbf{3}$ for the formation of the second block. In the calculations of the amount of monomer and initiator needed in order to obtain the desired percentages of both $(S)$ - and $(R)$-chiral monomer, the $(S)$-chiral thiophene, build in via initiator $\mathbf{5}$, has been taken into account.

(a)

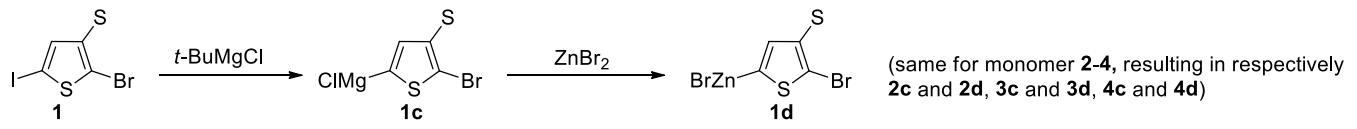

(b)
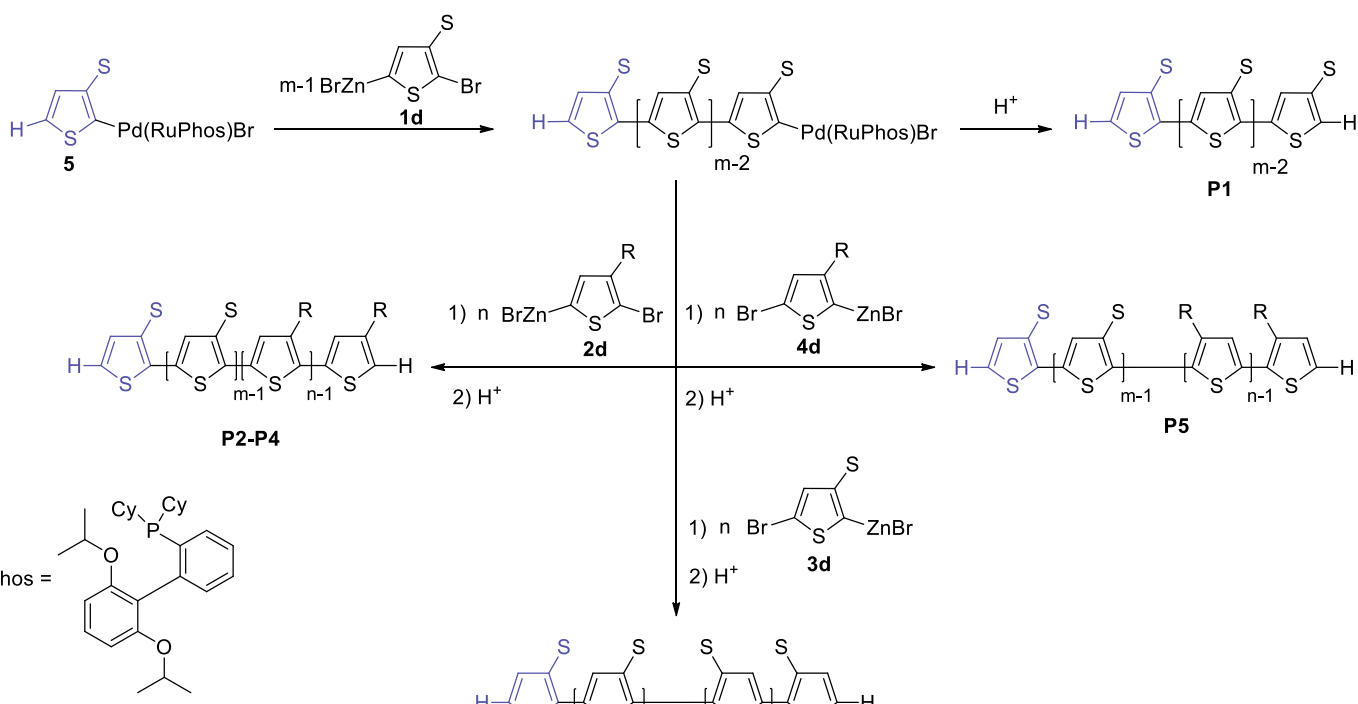

P1

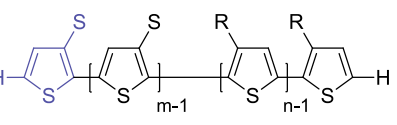

2-P4
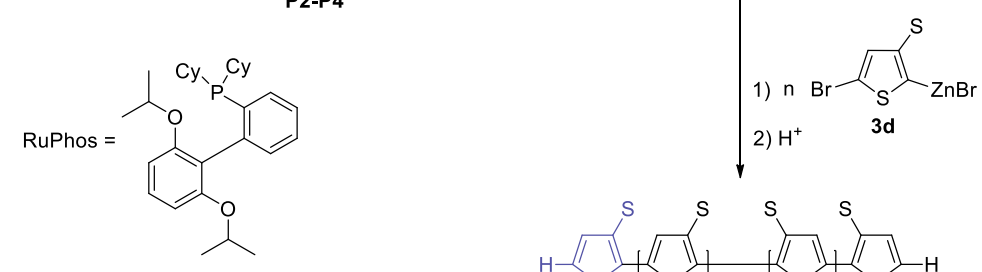

$\mathrm{Cy}=$ cyclohexane
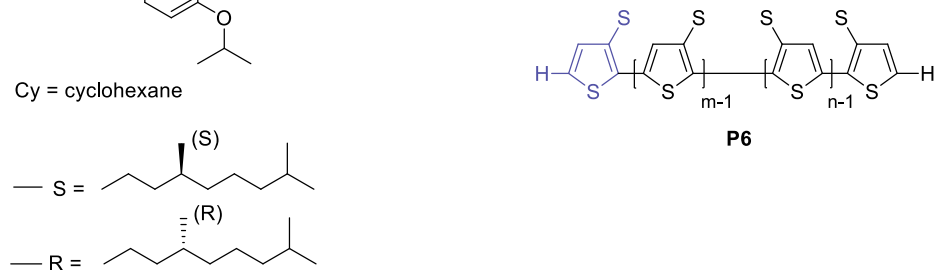

P6

Scheme 2. (a) Grignard metathesis and transmetalation of monomer 1-4; (b) Pd-RuPhos polymerization of P1-P6 with initiator 5.

Using gel permeation chromatography towards polystyrene standards, a first indication of a successful block copolymerization was obtained. The number average molar masses $\left(\bar{M}_{n}\right)$ and 
dispersities (Đ) of all synthesized polymers were determined using GPC (Table 1) as well as the degree of polymerization (DP) calculated from GPC data.

Table 1. Overview of $\bar{M}_{n}$ and Đ obtained by GPC, the DP calculated from GPC data and the DP $\mathrm{m}$ and $\mathrm{n}$ obtained by ${ }^{1} \mathrm{H}$ NMR.

$\begin{array}{ccccccc}\text { Polymer } & \bar{M}_{\boldsymbol{n}}\left(\mathbf{k g ~ m o l}^{-\mathbf{1}}\right) & \text { DP } & \text { D } & \text { m } & \text { n } & \%(S)-\%(R) \\ \text { P1 } & 4.2 & 19 & 1.5 & 18 & / & 100 \%(S)-0 \%(R) \\ \text { P2 } & 3.5 & 16 & 1.5 & 14 & 2 & 88 \%(S)-12 \%(R) \\ \text { P3 } & 3.6 & 16 & 1.4 & 12 & 4 & 75 \%(S)-25 \%(R) \\ \text { P4 } & 4.2 & 19 & 1.4 & 9 & 9 & 50 \%(S)-50 \%(R) \\ \text { P5 } & 4.8 & 22 & 1.3 & 11 & 11 & 50 \%(S)-50 \%(R) \\ \text { P6 } & 4.9 & 22 & 1.4 & 11 & 12 & 48 \%(S)-52 \%(S)\end{array}$

Although GPC gives an estimation of $\bar{M}_{n}$, the use of polystyrene standards inevitably results in a deviation in molar mass. However, end-group quantification via ${ }^{1} \mathrm{H}$ NMR provides the DP as well as the monomer ratio of both blocks $\left({ }^{1} \mathrm{H}\right.$ NMR spectra see supporting information S9S13). This was done via the assignment of the signals of the end-groups and internal monomer units, as shown in figure 3. Both aromatic and aliphatic region of the ${ }^{1} \mathrm{H}$ NMR were used, and showed matching results. For all first blocks isolated during the polymerization, as well as homopolymer P1 and block copolymers P2-P4 the DP was calculated with Eq. 1.

$$
D P=X-(1-Y)=\frac{V+W}{2}
$$

Eq. 1 
Although the aromatic region could be used for the determination of the DP of the first block of polymer P5 and P6, it could not be used for the block copolymers. This is due to the fact that the end-groups are exactly the same and the exact amount of Br-terminated ends is unknown. The Br-terminated polymer chains originate from dormant polymer chains. However, since the $\alpha$-methylene protons of the end-groups as well as of the internal $\mathrm{HH}$ coupled units form a separated signal from the other $\alpha$-methylene protons, it was possible to use the aliphatic ${ }^{1} \mathrm{H}$ NMR region (Figure 4). The DP was calculated using Eq. 2.

$$
D P=\frac{V+W}{2} \text { and } W=8
$$
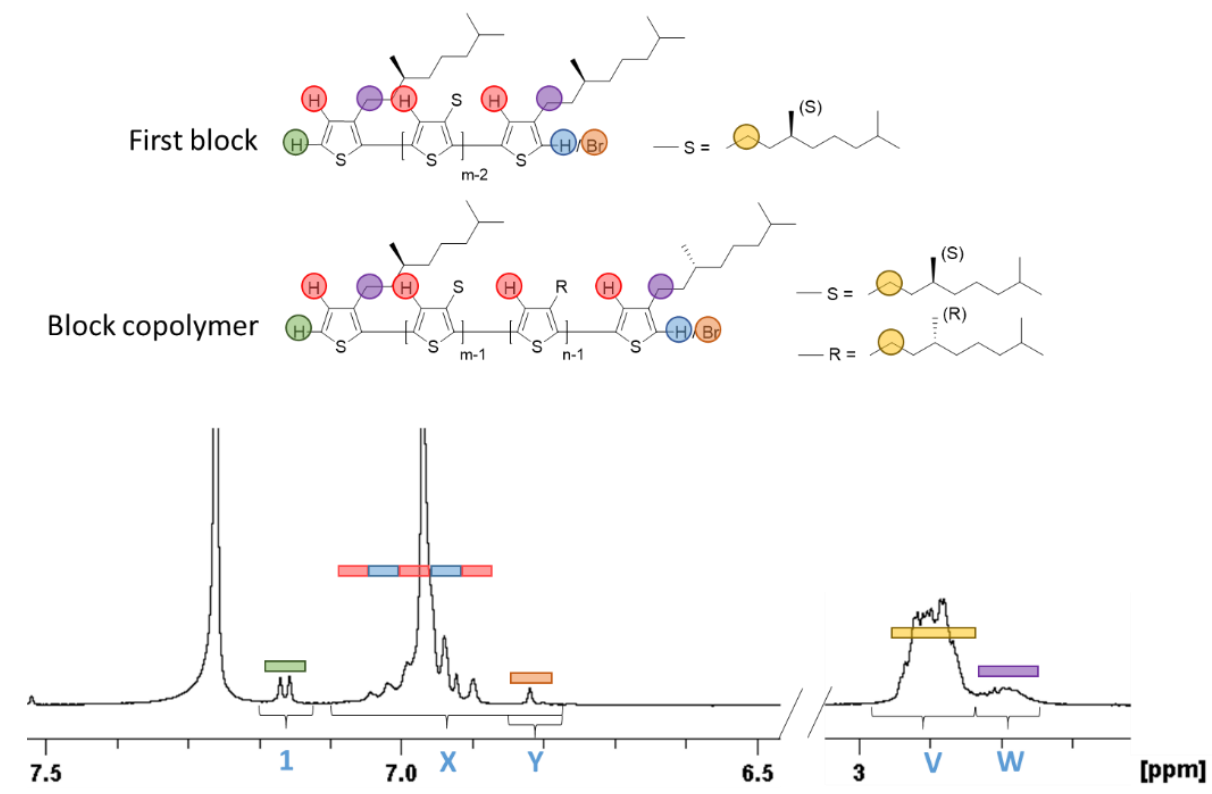

Figure 3. Assignment of ${ }^{1} \mathrm{H}$ NMR signals in the aromatic and aliphatic region for the first blocks isolated during the polymerization as well as homopolymer P1 and block copolymers

\section{P2-P4.}

When the DPs calculated from GPC data are compared to those obtained via ${ }^{1} \mathrm{H}$ NMR, surprisingly only negligible differences are obtained. 

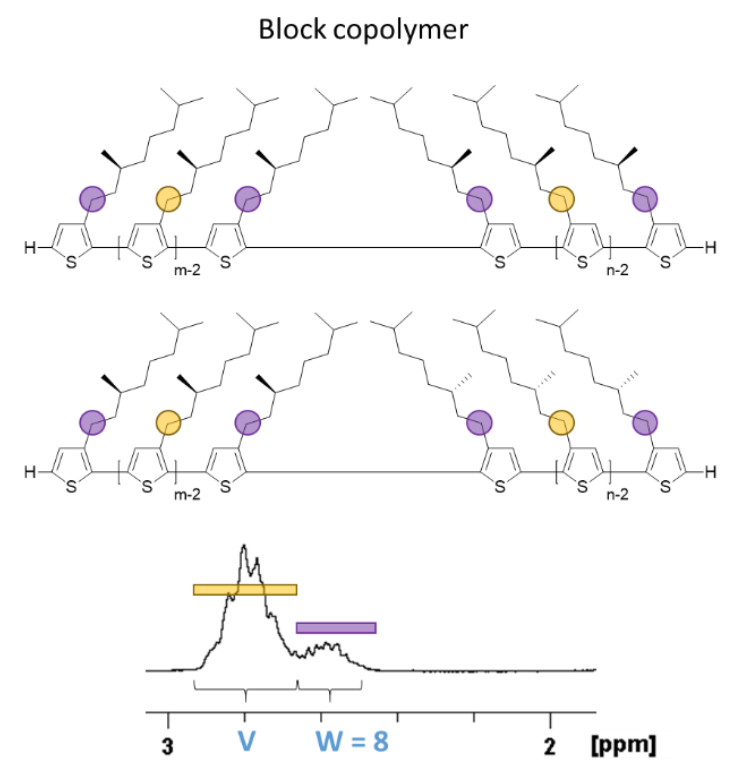

Figure 4. Assignment of ${ }^{1} \mathrm{H}$ NMR signals in the aromatic and aliphatic region for block copolymers P5 and P6.

Study of the chiral expression. In order to obtain a good insight into the chiral expression originating from the supramolecular organization, a solvatochromism experiment was performed for polymers P1-P6 and compared to P0, which has already been reported. ${ }^{29}$ During this solvatochroism experiment, the supramolecular organization of the different polymers was followed using UV-vis and CD spectroscopy. ${ }^{30-32}$ While UV-vis probes the formation of a supramolecular structure, $\mathrm{CD}$ visualizes the formation of chiral supramolecular structures. The experiment starts from a solution of the polymers in chloroform, a good solvent, to which methanol, a poor solvent, was gradually added. In order to minimize variations of the CD signal, the poor solvent was added with a constant speed of $0.20 \mathrm{~mL} / \mathrm{min}$. This was done via the use of a syringe pump. The most relevant UV-vis and CD results obtained for all the polymers are shown in figure 5 (all spectra can be found in supporting information S14-S20). Since the aggregation of the different polymers are qualitatively compared (does the polymer (chirally) aggregate or not) and not quantitatively (how well does the polymer aggregate / 
intensity of the CD signal) and regioregular PT with DP of 16 are still able to aggregate, the differences in DP between the polymers can be ignored.
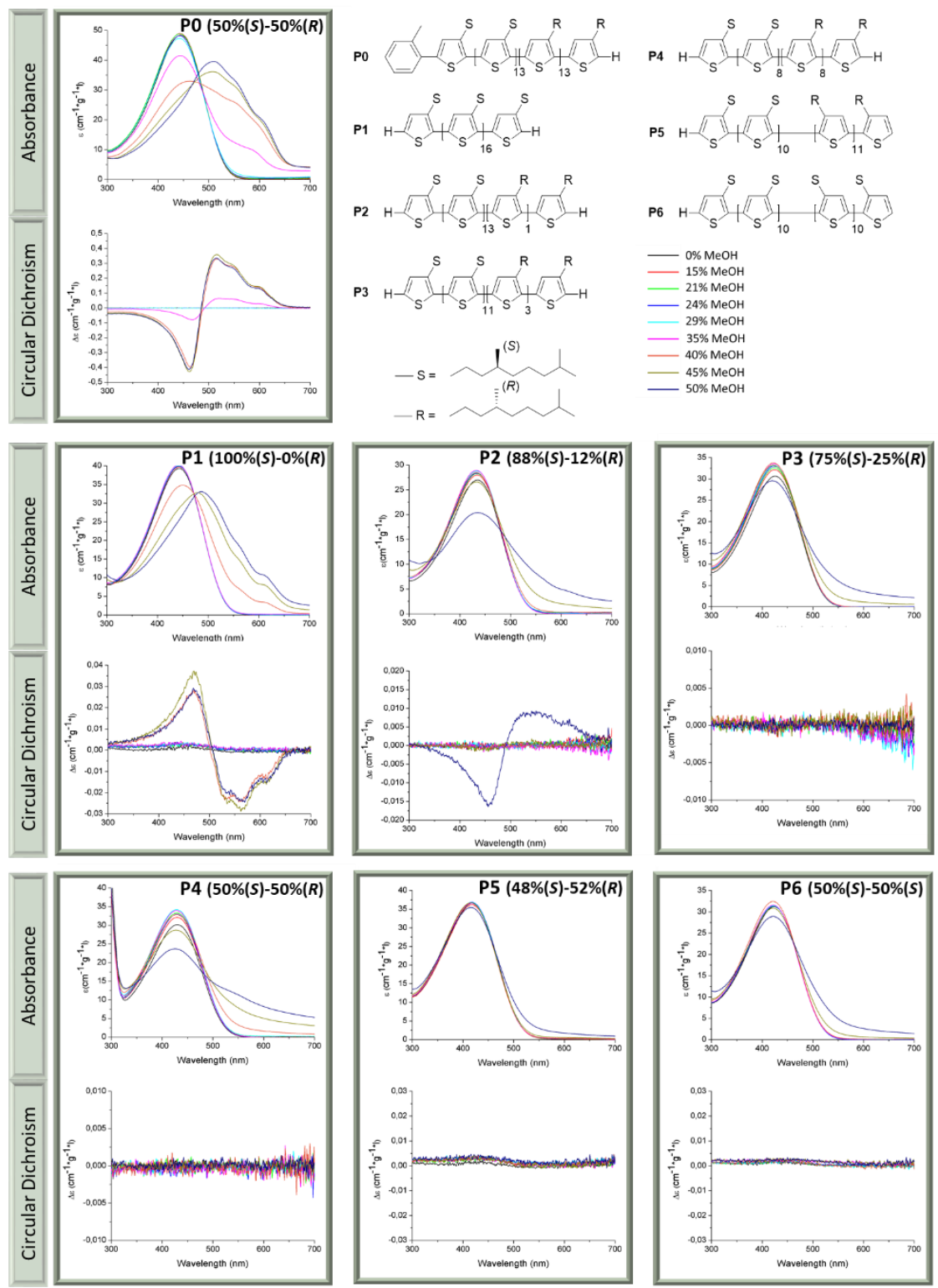

Figure 5. UV-vis and CD results of P0-P6, ${ }^{19}$ obtained via a solvatochroism experiment.

Upon addition of methanol, a red shift of the wavelength of maximum absorption $\left(\lambda_{\max }\right)$ as well as the appearance of fine structure around $620 \mathrm{~nm}$, typical for the aggregation of P3ATs, is visible in the UV-vis spectra of the homo $(S)$-chiral polymer P1. At low percentages of methanol no CD signal is visible. This is due to the fact that the polymer chains adopt a random coil-like structure, which is achiral. However, at a critical amount of methanol, 40\%, a bisignate 
Cotton-effect arise $\left(\mathrm{g}_{\mathrm{abs}}=\Delta \varepsilon / \varepsilon=1.1 \cdot 10^{-3}\right)$. More in particular, when chiral P3ATs start to organize, a one-handed helical (M- or P-helix) stack is formed, resulting in this typical bisignate signal (- or + ). For block copolymer P2, which contains $88 \%$ of $(S)$-chiral monomer and $12 \%$ of $(R)$-chiral monomer, the typical red shift as well as fine structure are almost not visible. Furthermore, the bisignate CD signal appears at a higher methanol content and is much weaker $\left(\mathrm{g}_{\mathrm{abs}}=8.0 \cdot 10^{-4}\right)$. These $\mathrm{UV}$-vis and CD results clearly show that polymer $\mathbf{P} 2$ had more difficulties to stack, in comparison to $\mathbf{P 1}$. Although $\mathbf{P 2}$ consist for $88 \%$ of $(S)$-chiral monomer, an opposite CD signal is obtained compared to $\mathbf{P 1}$ (which has already seen before) ${ }^{33}$ It can be speculated that block copolymers aggregate in a different way than homopolymers. ${ }^{19,33}$ Further, detailed research is currently performed using nonlinear optical techniques. For block copolymer P3 $(75 \%(S)-25 \%(R))$ and $\mathbf{P 4}(50 \%(S)-50 \%(R))$, no changes in the UV-vis spectra arise and no typical absorption around $620 \mathrm{~nm}$ is present. Also no CD signal is present, which is in line with the absence of aggregation. The results for this last polymer are surprising. Both block copolymers P0 and P4 possess no excess of chiral centers and have the same DP. The only difference between the two polymers is the presence of the $o$-tolyl end-group in $\mathbf{P 0}$. However, the gradually addition of poor solvent resulted in the formation of chiral aggregates for P0 - resulting in a high chiral response in CD $\left(\mathrm{g}_{\mathrm{abs}}=1.4 \cdot 10^{-2}\right)$ - while $\mathbf{P 4}$ did not organize at all. In previous research we concluded that the presence of the $o$-tolyl end-group is enough to create a difference in solubility between both blocks. This results in the aggregation of the $(R)$-chiral block, which imposed its chiral stacking to the later aggregating $(S)$-chiral block. However, since this end-group is not present in $\mathbf{P 4}$, both blocks behave in the same way. Neither the $(R)$-chiral block, nor the $(S)$-chiral block, was able to impose its handedness to the other block, resulting in a complete lack of supramolecular organization. Next, P5 was investigated. A chiral supramolecular organization is not anticipated as the polymer is achiral. Nevertheless, also here no supramolecular organization was obtained according to UV-vis 
spectroscopy. This can be due to the same reason as the non-aggregating behavior of $\mathbf{P 4}$ or due to the presence of the single HH-coupling, connecting both $(S)$ - and $(R)$-chiral block. In order to better understand the behavior of P5, the same, but homo $(S)$-chiral polymer $\mathbf{P 6}$ was investigated. Note that although P6 is a copolymer - the side chain is present at a different position and it is prepared as a copolymer (i.e. by sequential monomer addition) - it can be regarded as a homopolymer with a single $\mathrm{HH}$-defect. This polymer can be compared with P3ATs prepared from a Ni ${ }^{+I I}$ salt, which have a TT-defect. Although it is a homo $(S)$-chiral polymer, just as $\mathbf{P 1}$, no supramolecular organization was obtained. From these results we can conclude that the non-aggregating behavior of P5 and P6 originates from the presence of a single HH-coupling in the middle of the polymer chain. This is in contrast with the strong CD effect found in chiral P3AT with a TT-defect. ${ }^{21}$

In order to validate the results obtained with UV-vis spectroscopy and $\mathrm{CD}$, differential scanning calorimetry (DSC) was recorded for all polymers (supporting information S21-S24). The absence of melting and crystallization peaks for polymer P3-P6, and thus supramolecular organization, confirms the findings from UV-vis spectroscopy and CD. Since polymers may be able to crystallize very slowly, the prepared samples of the polymers were left to equilibration and again recorded one week later (supporting information S21-S24). Since the same results were obtained we can conclude that the absence of an end-group as well as the presence of one $\mathrm{HH}$-coupling results in a non-aggregating behavior.

\section{CONCLUSION}

In this manuscript, supramolecular organization and chiral expression of P3ATs are examined. The polymers were prepared using Pd-RuPhos protocol and were analyzed by GPC and ${ }^{1} \mathrm{H}$ NMR. Solvatochromism experiments revealed two important molecular influences on the supramolecular organization and chiral expression. First, in block copolymers consisting of an 
$(S)$-chiral and $(R)$-chiral block of equal length, end-groups are required to break the symmetry. If no end-group is present, the polymer chains do not aggregate. Second, already one single $\mathrm{HH}$-coupling disrupts the supramolecular aggregation in regioregular polythiophene, which is in contrast with the presence of one TT-defect.

\section{AUTHOR INFORMATION}

\section{Corresponding author}

*E-mail guy.koeckelberghs @kuleuven.be (G.K.).

\section{Notes}

The authors declare no competing financial interest.

\section{ACKNOWLEDGEMENT}

We are grateful to the Onderzoeksfonds KU Leuven/Research Fund KU Leuven for funding this project.

\section{ABBREVIATIONS}

CP, Conjugated Polymers; GPC, Gel Permeation Chromatography; CD, Circular Dichroism; HH, Head-Head; HT, Head-Tail; TT, Tail-Tail; DSC, Differential Scanning Calorimetry; P3AT, poly(3-alkylthiophene); PT, poly(thiophene); dppp, bisdiphenylphosphinopropane; dppe, bisdiphenylphosphinoethane; KCTCP, Kumada Catalyst Transfer Condensative Polymerization; GRIM, Grignard Metathesis; THF, Tetrahydrofurane; MeOH, methanol; Đ, Dispersity; $\bar{M}_{n}$, Number average molar mass; DP, Degree of Polymerization; $\varepsilon$, extinction coefficient; $\Delta \varepsilon$, difference in extinction coefficient of left and right circularly polarized light; $\mathrm{g}_{\text {abs, }}$ anisotropic factor; $\lambda_{\max }$, wavelength of maximum absorption. 


\section{ASSOCIATED CONTENT}

Supporting Information. Used instrumentation and experimental details as well as ${ }^{13} \mathrm{C}$ NMR, UV-vis spectra, CD spectra and DSC spectra.

\section{REFERENCES}

(1) Jonoska, N.; Seeman, N. C. Computing by Molecular Self-Assembly. Interface Focus 2012, 2 (4), 504-511.

(2) Kaushik, M.; Sinha, P.; Jaiswal, P.; Mahendru, S. Protein Engineering and de Novo Designing of a Biocatalyst. 2016, 29, 499-503.

(3) Jones, M. R.; Seeman, N. C.; Mirkin, C. A. Programmable Materials and the Nature of the DNA Bond. Science 2015, 347, 12609011-126090111.

(4) Huang, J.; Ding, H. M.; Xu, Y.; Zeng, D.; Zhu, H.; Zang, D. M.; Bao, S. S.; Ma, Y. Q.; Zheng, L. M. Chiral Expression from Molecular to Macroscopic Level via $\mathrm{pH}$ Modulation in Terbium Coordination Polymers. Nat. Commun. 2017, 8 (1), 1-12.

(5) Asakawa, M.; Brancato, G.; Fanti, M.; Leigh, D. A.; Shimizu, T.; Slawin, A. M. Z.; Wong, J. K. Y.; Zerbetto, F.; Zhang, S. Switching "on" and "off” the Expression of Chirality in Peptide Rotaxanes. J. Am. Chem. Soc. 2002, 124 (12), 2939-2950.

(6) Wu, T.; You, X. Z.; Bouř, P. Applications of Chiroptical Spectroscopy to Coordination Compounds. Coord. Chem. Rev. 2015, 284, 1-18.

(7) Wu, L.; Wang, Q.; Lu, J.; Bian, Y.; Jiang, J.; Zhang, X. Helical Nanostructures SelfAssembled from Optically Active Phthalocyanine Derivatives Bearing Four Optically Active Binaphthyl Moieties: Effect of Metal-Ligand Coordination on the Morphology, Dimension, and Helical Pitch of Self-Assembled Nanostructures. Langmuir 2010, 26 
(10), 7489-7497.

(8) Liu, Y.; Tang, Z. Nanoscale Biocoordination Polymers: Novel Materials from an Old Topic. Chem. - A Eur. J. 2012, 18 (4), 1030-1037.

(9) Fuhr, J. D.; Van der Meijden, M. W.; Cristina, L. J.; Rodríguez, L. M.; Kellogg, R. M.; Gayone, J. E.; Ascolani, H.; Lingenfelder, M. Chiral Expression of Adsorbed (MP) 5amino[6]helicenes: From Random Structures to Dense Racemic Crystals by Surface Alloying. Chem. Commun. 2017, 53 (1), 130-133.

(10) Wu, P. T.; Ren, G.; Kim, F. S.; Chaoxu, L. I.; Raffaele, M.; Jenekhe, S. A. Poly(3Hexylthiophene)-B-poly(3-Cyclohexylthiophene): Synthesis, Microphase Separation, Thin Film Transistors, and Photovoltaic Applications. J. Polym. Sci. Part A Polym. Chem. 2010, 48 (3), 614-626.

(11) Traina, C. A.; Charles, R.; Ii, B.; Bazan, G. C. Design and Synthesis of MonoFunctionalized, Water Soluble Conjugated Polymers for Biosensing and Imaging Applications. J. Am. Chem. Soc. 2011, 133, 12600-12607.

(12) Kim, J.; Song, I. Y.; Park, T. Polymeric Vesicles with a Hydrophobic Interior Formed by a Thiophene-Based All-Conjugated Amphiphilic Diblock Copolymer. Chem. Commun. 2011, 47 (16), 4697.

(13) Senkovskyy, V.; Tkachov, R.; Beryozkina, T.; Komber, H.; Oertel, U.; Horecha, M.; Bocharova, V.; Stamm, M.; Gevorgyan, S. A.; Krebs, F. C.; Kiriy, A. “ Hairy ” Poly ( 3-Hexylthiophene ) Particles Prepared via Surface-Initiated Kumada Catalyst-Transfer Polycondensation. J. Am. Chem. Soc. 2009, 131, 16445-16453.

(14) Oh, J. Y.; Shin, M.; Lee, T. Il; Jang, W. S.; Lee, Y. J.; Kim, C. S.; Kang, J. W.; Myoung, J. M.; Baik, H. K.; Jeong, U. Highly Bendable Large-Area Printed Bulk Heterojunction 
Film Prepared by the Self-Seeded Growth of poly(3-Hexylthiophene) Nanofibrils. Macromolecules 2013, 46 (9), 3534-3543.

(15) Willot, P.; Teyssandier, J.; Dujardin, W.; Adisoejoso, J.; De Feyter, S.; Moerman, D.; Leclère, P.; Lazzaroni, R.; Koeckelberghs, G. Direct Visualization of Microphase Separation in Block copoly(3-Alkylthiophene)s. RSC Adv. 2015, 5 (12), 8721-8726.

(16) Tsai, C.; Fortney, A.; Qiu, Y.; Gil, R. R.; Yaron, D.; Kowalewski, T.; Noonan, K. J. T. Conjugated Polymers with Repeated Sequences of Group 16 Heterocycles Synthesized through Catalyst-Transfer Polycondensation. J. Am. Chem. Soc. 2016. 138, 6798-6804.

(17) Monnaie, F.; Ceunen, W.; De Winter, J.; Gerbaux, P.; Cocchi, V.; Salatelli, E.; Koeckelberghs, G. Synthesis and Transfer of Chirality in Supramolecular Hydrogen Bonded Conjugated Diblock Copolymers. Macromolecules 2015, 48 (1), 90-98.

(18) Monnaie, F.; Van Den Eede, M. P.; Koeckelberghs, G. Expression of Chirality in a Conjugated Polymer without Any Excess of Chiral Centers. Macromolecules 2015, 48 (22), 8121-8127.

(19) Van Den Eede, M.-P.; Bedi, A.; Delabie, J.; De Winter, J.; Gerbaux, P.; Koeckelberghs, G. The Influence of the End-Group on the Chiral Self-Assembly of All-Conjugated Block Copolymers. Polym. Chem. 2017, 8 (37), 5666-5672.

(20) Verswyvel, M.; Monnaie, F.; Koeckelberghs, G. AB Block copoly(3-Alkylthiophenes): Synthesis and Chiroptical Behavior. Macromolecules 2011, 44 (24), 9489-9498.

(21) Willot, P.; Steverlynck, J.; Moerman, D.; Leclère, P.; Lazzaroni, R.; Koeckelberghs, G. Poly(3-Alkylthiophene) with Tuneable Regioregularity: Synthesis and Self-Assembling Properties. Polym. Chem. 2013, 4 (9), 2662-2671. 
(22) Senkovskyy, V.; Khanduyeva, N.; Komber, H.; Oertel, U.; Stamm, M.; Kuckling, D.; Kiriy, A. Conductive Polymer Brushes of Regioregular Head-to-Tail poly(3Alkylthiophenes) via Catalyst-Transfer Surface-Initiated Polycondensation. J. Am. Chem. Soc. 2007, 129 (20), 6626-6632.

(23) Khanduyeva, N.; Senkovskyy, V.; Beryozkina, T.; Bocharova, V.; Simon, F.; Nitschke, M.; Stamm, M.; Grötzschel, R.; Kiriy, A. Grafting of poly(3-Hexylthiophene) from poly(4-Bromostyrene) Films by Kumada Catalyst-Transfer Polycondensation: Revealing of the Composite Films Structure. Macromolecules 2008, 41 (20), 73837389.

(24) Doubina, N.; Ho, A.; Jen, A. K. Y.; Luscombe, C. K. Effect of Initiators on the Kumada Catalysttransfer Polycondensation Reaction. Macromolecules 2009, 42 (20), 76707677.

(25) Bronstein, H. A.; Luscombe, C. K. Externally Initiated Regioregular P3HT with Controlled Molecular Weight and Narrow Polydispersity. J. Am. Chem. Soc. 2009, 131 (36), 12894-12895.

(26) Kaul, E.; Senkovskyy, V.; Tkachov, R.; Bocharova, V.; Komber, H.; Stamm, M.; Kiriy, A. Synthesis of a Bifunctional Initiator for Controlled Kumada Catalyst-Transfer Polycondensation/nitroxide-Mediated Polymerization and Preparation of poly(3Hexylthiophene)-Polystyrene Block Copolymer Therefrom. Macromolecules 2010, 43 (1), 77-81.

(27) Wu, S.; Huang, L.; Tian, H.; Geng, Y.; Wang, F. LiCl-Promoted Chain Growth Kumada Catalyst-Transfer Polycondensation of The "reversed" thiophene Monomer. Macromolecules 2011, 44 (19), 7558-7567. 
(28) Verswyvel, M.; Verstappen, P.; De Cremer, L.; Verbiest, T.; Koeckelberghs, G. Development of a Universal Chain-Growth Polymerization Protocol of Conjugated Polymers: Toward a Variety of All-Conjugated Block-Copolymers. J. Polym. Sci. Part A Polym. Chem. 2011, 49 (24), 5339-5349.

(29) Van Den Eede, M.-P.; Bedi, A.; Delabie, J.; De Winter, J.; Gerbaux, P.; Koeckelberghs, G. The Influence of the End-Group on the Chiral Self-Assembly of All-Conjugated Block Copolymers. Polym. Chem. 2017, 8 (37), 5666-5672.

(30) Langeveld-Voss, B. M. W.; Christiaans, M. P. T.; Janssen, R. A. J.; Meijer, E. W. Inversion of Optical Activity of Chiral Polythiophene Aggregates by a Change of Solvent. Macromolecules 1998, 31 (19), 6702-6704.

(31) Langeveld-Voss, B. M. W.; Beljonne, D.; Shuai, Z.; Janssen, R. A. J.; Meskers, S. C. J.; Meijer, E. W.; Brødas, J. Investigation of Exciton Coupling in Oligothiophenes by Circular Dichroism Spectroscopy. Adv. Mater. 1998, 10 (16), 1343-1348.

(32) Lermo, E. R.; Langeveld-voss, B. M. W.; Janssen, R. A. J.; Meijer, E. W. Odd - Even Effect in Optically Active Poly ( 3 , 4-Dialkoxythiophene ). Chem. Commun. 1999, 791792.

(33) Bidan, G.; Guillerez, S.; Sorokin, V. Chirality in Regioregular and Soluble Polythiophene: An Internal Probe of Conformational Changes Induced by Minute Solvation Variation. Adv. Mater. 1996, 8 (2), 157-160. 


\section{FOR TABLE OF CONTENTS ONLY}

Expression of Chirality in Tailor-made Conjugated Polymers

Marie-Paule Van Den Eede ${ }^{1}$, Lore Van Gestel ${ }^{1}$ and Guy Koeckelberghs ${ }^{1 *}$

${ }^{1}$ Laboratory for Polymer Synthesis, KU Leuven, Celestijnenlaan 200F, B-3001 Heverlee, BELGIUM

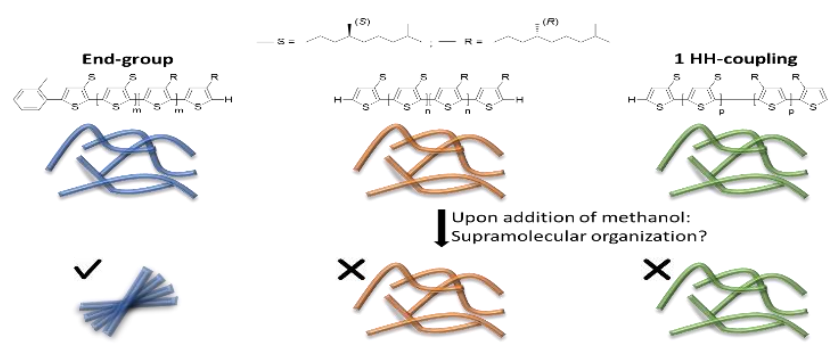

